\title{
Roles of Orientation in Tabletop Collaboration: Comprehension, Coordination and Communication
}

\author{
RUSSELL KRUGER, SHEELAGH CARPENDALE, STACEY D. SCOTT \\ \& SAUL GREENBERG \\ Department of Computer Science, University of Calgary, 2500 University Drive NW, Calgary, \\ Alberta, Canada T2N 1N4 (E-mail: \{krugerj, sheelagh, sdscott, saul\}@ucalgary.ca)
}

\begin{abstract}
In order to support co-located collaboration, many researchers are now investigating how to effectively augment tabletops with electronic displays. As far back as 1988, orientation was recognized as a significant human factors issue that must be addressed by electronic tabletop designers. As with traditional tables, when people stand or sit at different positions around a horizontal display they will be viewing the contents from different angles. One common solution to this problem is to have the software reorient objects so that a given individual can view them 'right way up.' Yet is this the best approach? If not, how do people actually use orientation on tables? To answer these questions, we conducted an observational study of collaborative activity on a traditional table. Our results show that the strategy of reorienting objects to a person's view is overly simplistic: while important, it is an incomplete view of how people exploit their ability to reorient objects. Orientation proves critical in how individuals comprehend information, how collaborators coordinate their actions, and how they mediate communication. The coordinating role of orientation is evident in how people establish personal and group spaces and how they signal ownership of objects. In terms of communication, orientation is useful in initiating communicative exchanges and in continuing to speak to individuals about particular objects and work patterns as collaboration progresses. The three roles of orientation have significant implications for the design of tabletop software and the assessment of existing tabletop systems.
\end{abstract}

Key words: collaborative computing, co-located collaboration, computer-supported cooperative work, interface design, observational study, orientation, rotation, synchronous interaction, tabletop display

\section{Introduction}

When people collaborate in face-to-face settings, they often use tools such as pens, pencils, paper, and printouts, and work over some sort of supporting surface or table. They share information placed on this surface, use objects on the table as conversational props, or develop ideas and work products (Tang 1991; Brinck and Gomez, 1992). In contrast, desktop computers may inhibit the group interaction, even though they potentially allow access to 
information and productivity tools pertinent to the task at hand. For example, adults are forced to sit closer than is socially comfortable (Hall, 1966), and people resort to a turn-taking interaction style because they cannot interact in their typically synchronous manner (Scott et al., 2002). In fact, Luff et al. (1992) found that desktop computers are often abandoned for traditional tools during co-located collaboration.

Many researchers are now interested in combining the advantages of computers and tables through the development of tabletop displays. The technology is nearly in place: high quality projectors, flat panel and plasma displays, and touch-sensitive surfaces mean that it is now fairly straight-forward to construct a tabletop display. The challenge is one of design: electronic tables should at least maintain those characteristics of traditional tables that prove particularly useful for collaboration, such as awareness of other participants' actions, the possibility of simultaneous interaction, and affordances for sharing (Kruger et al., 2002). Of course, there are restrictions imposed by physical tables that electronic ones may mitigate or bypass altogether. As with many computer applications, they can provide the group with powerful tools and interaction techniques, as well as access to information and data that go far beyond what is normally available on a physical surface. However, these tools and interaction techniques must be appropriate; otherwise they will interfere with the group process.

One seemingly small but important aspect of electronic tabletop design is orientation, i.e., how the angle of objects are positioned relative to the participants seated around the table. As with traditional tables, when people work at different positions around a horizontal display they will be viewing its contents from different angles. For example, when two people are seated at different sides of a table, an object that is 'right way up' to one will appear upside down to the other. When pertinent information is upside down or sideways, people may have more difficulty understanding it, or may misinterpret actions performed by others on that information.

Currently, we do not really know how people use orientation as they work together over a workspace. Without this knowledge, designing new interaction techniques for orientation will be a hit-and-miss exercise, or even worse, may hinder the collaborative group processes if the design approach is wrong. It is this issue of object orientation and its role in collaboration that is the focus of this paper.

Our goal is to understand how the orientation of artifacts affects collaboration on a horizontal workspace and the implications this has for the design of collaborative tabletop displays. This paper expands our previous introduction of the roles of orientation (Kruger et al., 2003) by presenting additional details of the methodology and data from an observational study of tabletop collaboration which significantly influenced our understanding of 
the subtleties of tabletop orientation. We also include an updated evaluation of orientation techniques used in current tabletop systems.

In Section 2, we describe how existing designs of tabletop systems address orientation. Then, based on the aforementioned observational study and a subsequent video analysis of this study, we identify and discuss three roles that orientation plays in the collaborative process: comprehension, coordination, and communication. Finally, we discuss the implications these roles of orientation have for the design and evaluation of tabletop interfaces.

\section{Related work}

Previous tabletop display systems use one of several basic approaches to handle orientation.

Fixed orientation. Some systems assume a single orientation, and that participants will sit side-by-side instead of on different sides of the table. An example is the Café Table (de Bruijn and Spence, 2001; Stathis et al., 2002), where a small semi-circular electronic display embedded in one end of an oval espresso table is configured for side-by-side seating. The main workspace of the table display aligns all items to a front-facing, fixed orientation. A variation of fixed orientation is provided by applications built atop the DiamondSpin Java toolkit, which is used for creating tabletop interfaces (Shen et al., 2004). DiamondSpin provides the optional functionality to temporarily adjust all items in a central workspace to a global alignment. For example, a person using the Personal Digital Historian application (built atop DiamondSpin) can invoke a "Magnet" tool by pressing a Magnet toggle button on his or her personal toolbar to reorient all items in the central workspace to face the Magnet button (Vernier et al., 2002). When the Magnet tool is toggled off, items are automatically returned to their previous orientation.

Manual orientation. The conceptually simplest way to manage orientation is to let users manually rotate their own information items on the tabletop. This is, of course, a direct analog of how people now interact with traditional media on a table, such as paper. While people have considerable experience rotating traditional media, manually rotating digital objects can be more difficult. Rotation is clumsy in electronic settings, likely because current input devices (e.g., mouse, keyboard, and stylus) provide few degrees of freedom compared to the easy manipulations possible with one's hand on a physical object. Extra controls are typically needed, such as the rotation mode and "handle" provided by Microsoft PowerPoint ${ }^{\circledR}$ for free rotation, or a menu to rotate an object a set amount.

To facilitate manual orientation, several tables with pen-based input now provide users with lightweight rotation mechanisms, such as the circular pen 
gesture employed by the InteracTable (Streitz et al., 1999, 2001) and the ConnecTable (Streitz et al., 2001; Tandler et al., 2001). The DiamondSpin toolkit (Shen et al., 2004) provides application developers the option of adding a rotation handle to the bottom-left corner of tabletop objects to enable manual rotation. This rotation handle allows users to reorient tabletop items, such as digital documents (Ringel et al., 2004) and digital photos (Shen et al., 2004), for personal use or for sharing with others.

Multiple copies. One way of finessing the orientation problem is to give each person their own copy of tabletop objects, which they can orient any way they wish. In the ConnecTable, people join two small personal tables together to form a larger homogeneous display for face-to-face work (Streitz et al., 2001; Tandler et al., 2001). One mode of operation involves multiple copies of information, where each individual has a personal copy of a shared object on his or her side of the table. This concept is generalized in the underlying BEACH architecture (Streitz et al., 2001), used by the InteracTable, that lets multiple copies of an object be maintained in the shared workspace. These copies can each be positioned independently; therefore, copies can be oriented in different directions to support group members located at different sides of the table. All copies share the same underlying representation of the object; thus, changes made to one copy of the object are reflected in all copies of the object.

Person-based automatic orientation. A few designers have tried to minimize the need for manual rotations by having the system automatically orient information items on the tabletop workspace. In the person-based approach, information is oriented towards the person who has most recently accessed the information. This strategy assumes that the person manipulating the item benefits most from the 'best view' of the information. For example, people seated at the InfoTable (Rekimoto and Saitoh, 1999) can access items on the tabletop display by using the pointing device on their respective laptops (also located on the table). When a person drags a tabletop item to "their" side of the table, the item automatically rotates toward the table edge closest to their laptop.

Person-based automatic orientation is also used by Magerkurth et al. (2003) in the STARS augmented tabletop board game. Interaction in the game is turn-based, and during each turn the electronic game board and any invoked menu items are automatically oriented towards the currently active player. To our understanding, the location and number of players are currently predefined in the STARS application, but computer vision techniques or user-aware tabletop hardware, such as the DiamondTouch system (Deitz and Leigh, 2001), could also be used with a turn-based strategy to allow more flexibility for joining and leaving the game.

Finally, the ConnecTable (Streitz et al., 2001; Tandler et al., 2001) has another mode of operation that automatically rotates objects as they are 
moved across the seam between the two small connected surfaces. If a person on one side drags an object to the other side, the object automatically rotates by $180^{\circ}$, which orients it right-side up for the receiving person.

Environment-based automatic orientation. A limitation of the person-based approach is that tabletop systems often do not know exactly where the person manipulating the item is located; thus, the information may be automatically oriented towards the wrong side of the table. This problem may be solved soon, for several new technologies now provide more accurate person-location detection (Deitz and Leigh, 2001; Hancock and Booth, 2004). Still, some have questioned whether it is always appropriate to orient the information towards the person manipulating the information, for appropriateness often depends on the intent of the manipulation. Thus, an environment-based approach has been proposed by other researchers, where the system orients information based on its location in the tabletop environment.

The environment-based strategy typically assumes that the person who is closest to the information - regardless of who has selected it - would be the one most likely to benefit from the 'best view' of the information. For example, the DiamondSpin toolkit (Shen et al., 2004) enables automatic adjustment of digital items towards the outside of the tabletop workspace. Therefore, any item directly in front of a person will always be oriented towards that person, regardless of who is manipulating it. The Personal Digital Historian (Shen et al., 2001, 2002; Vernier et al. 2002), the UbiTable (Shen et al., 2003), and the Opportunistic Browsing Table (Shen et al., 2004), systems all built atop DiamondSpin, use this feature to automatically align digital photos and documents towards the outer edge of a central group workspace. In addition, the UbiTable system provides group members personal workspaces on the table, located against the table edges. When a person moves a document into his or her personal space, that document is automatically reoriented to be 'right way up' for them.

Similarly, icons on the perimeter of the Café Table (de Bruijn and Spence, 2001; Stathis et al., 2002) flow along the edge automatically, and as they flow, they are oriented tangentially to the table edge (although as stated previously, the main display has a fixed orientation). The InteracTable system automatically rotates objects as a consequence of a person "tossing" an information item to the other side of the table using a pen gesture. As the item moves across the table, it automatically rotates until it stops on the other side of the table, fully oriented towards the closest table edge (Streitz et al., 1999, 2001).

It is unclear which, if any, of these approaches suffice. There is relatively little prior work on orientation in tabletop interaction. Thus there is no basis to inform decisions about how best to present orientation-dependent information (e.g., text, menus, and icons) to collaborators working at a table. Existing design assumptions are likely too simplistic. For example, 
automated orientation mechanisms assume that readability of the item is critical. However, one of the few observational studies that considered orientation in collaborative tabletop interactions suggests otherwise. In particular, while Tang (1991) noted the familiar problems of information at odd angles causing reading and annotating difficulties, he also noted conditions where variant orientation served as a collaborative resource. For instance

- using someone else's orientation alignment conveyed support,

- orientation could be used to help establish an intended audience,

- orientation could be used to help establish one's personal space.

Another exploratory study, performed by Fitzmaurice et al. (1999), investigated the manipulation of artwork during the drawing process. Large variations were found in drawing styles between participants. Yet all participants used variant (i.e., non-orthogonal) orientation of their drawing canvas and many continued to vary the orientation of the canvas as they worked. Rotation of the canvas appeared to be performed for ergonomic (e.g., comfort), performance, and comprehensive (e.g., evaluative) reasons during both the writing and drawing tasks performed by their participants. While their focus was on supporting a single user drawing on a pen-based tablet-style computer, many of their recommendations may be applicable to handling orientation issues during tabletop collaboration. In particular, they discuss the need for full variation in orientation angle, and suggest the use of self-rotating and self-orienting user interaction elements.

\section{The impact of orientation on collaboration}

Previous person-based and environment-based approaches assume that the most significant orientation issue is that of readability (i.e., comprehension). These approaches assume that in designing tabletop interfaces, the critical question to address is how to know whom an object should be oriented towards at any given moment. Intrigued by Tang's observations and motivated by the importance of resolving this issue for the design of our own tabletop interfaces, we set out to improve our understanding of the roles that orientation can play in collaboration. We began a series of explorations into the impact of orientation on collaborative activities and interaction in general. These explorations involved gathering data from a variety of sources including prior art

- HCI and CSCW literature describing existing digital tabletop systems, (e.g., Rekimoto and Saitoh, 1999; Streitz et al., 1999, 2001; de Bruijn and 
Spence, 2001; Deitz and Leigh, 2001; Hancock and Booth, 2004; Shen et al., 2001, 2002; Tandler et al., 2001; Stathis et al., 2002; Vernier et al., 2002),

- HCI and CSCW literature involving qualitative and quantitative studies focused on tabletop collaboration or single-user tabletop interaction, (e.g., Tang, 1991; Fitzmaurice, et al., 1999).

Since no body of work had tackled orientation issues directly, we also conducted our own formative investigations. These included pilot studies, involving a series of design sessions on the granularity of rotation and variations in angular freedom (Kruger and Carpendale, 2003), and an observational study of collaboration on displays of various orientations (Kruger and Carpendale, 2002). Analyses of these formative studies revealed that rotation and orientation have complex and subtle effects on the process of tabletop collaboration. In order to further understand these effects, we performed an in-depth video analysis of one condition of the observational study which involved tabletop collaboration using traditional paper-based media. The following two sections briefly describe the observational study and the subsequent video analysis.

\section{The observational study}

The observational study was originally designed to compare and analyze how people collaborate over physical vs. electronic tables, and on upright vs. horizontal displays. While the study goals go beyond orientation (e.g., the research questions and study tasks involved issues other than orientation), we were able to use the same data for deeper analysis of orientation effects once we realized the key role orientation played in participant interaction. In this section, we describe the entire study setup. However, we narrow our description of the data analysis to only those study aspects that deal with orientation.

\subsection{EXPERIMENTAL SETUP}

The study involved dyads assembling a puzzle in three settings: (a) a traditional table setting, (b) a digital tabletop setting, and (c) an upright display setting (Figure 1). The traditional table was a $30 \times 49$ in. office table; the digital tabletop was created from a 20 in. LCD placed horizontally in a table approximately 30 inches wide, and the vertical display was a 21 in. flat-screen monitor. The resolution and actual usable size of the two display screens was equivalent $(1600 \times 1200)$. Both computerized settings used a Pentium IIIbased PC running Windows, and provided concurrent, multi-user interaction through the use of modified mice (Tse and Greenberg, 2002). We used mice rather than a touch sensitive surface (e.g., Deitz and Leigh, 2001) simply 


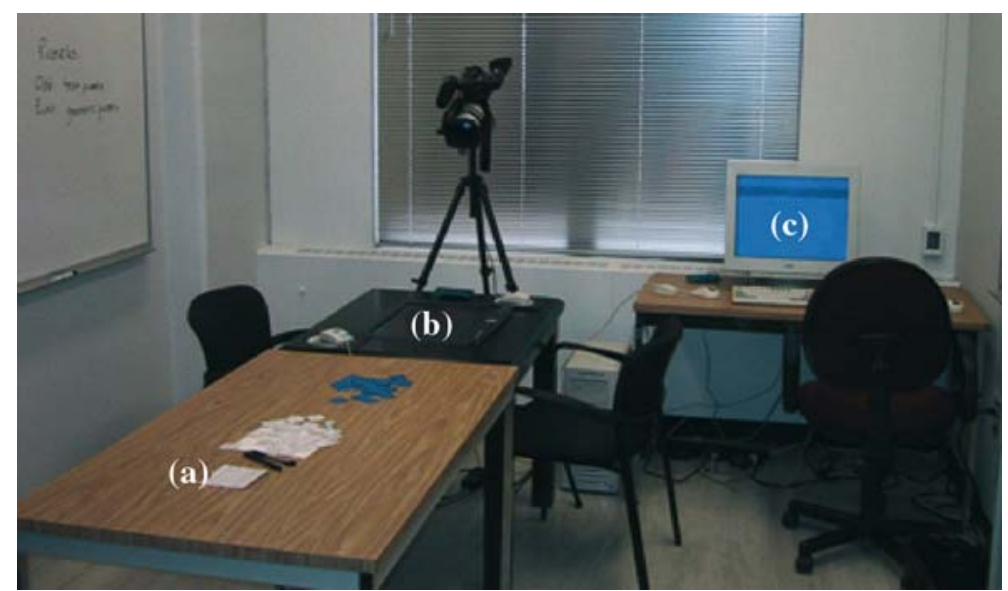

Figure 1. Experimental setup showing (a) the regular tabletop condition; (b) the digital tabletop condition; and (c) the upright display condition.

because a multiple-touch display was not available to us at the time of the study.

\subsection{THE TASK}

During the study, participants collaboratively assembled a jigsaw-type puzzle. We chose the puzzle task as a puzzle is often completed by groups of individuals, is typically done on horizontal surfaces, and allowed analysis of orientation as a variable of interest. As well, puzzle assembly shares attributes common to many collaborative construction tasks performed over tabletops. For instance, it involves a single shared artefact that is the "product" (i.e., the puzzle). It involves individual components that are manipulated for personal work (i.e., an individual's current puzzle pieces), and also involves objects intended for joint action (i.e., the puzzle-inprogress, as well as shared puzzle pieces).

We created an electronic puzzle application for the vertical and tabletop display conditions, and an equivalent paper-based puzzle for the nonelectronic tabletop condition. Several puzzles were created, some of which were geometric and essentially non-orientated (i.e., they did not contain any orientation cues to indicate one alignment as 'right way up'), and some were textbased with a clear top and bottom. Each puzzle consisted of 25 rectangular puzzle pieces which were positioned side-by-side to form a final image (Figures 2 and 3). For the electronic version, the completed puzzle rested inside the inner black square (Figure 2), and a preview image of the completed puzzle appeared to the right of the black square. Each puzzle piece was capable of being rotated $90^{\circ}$ by right-clicking on the piece. Support for two mice was included in the 


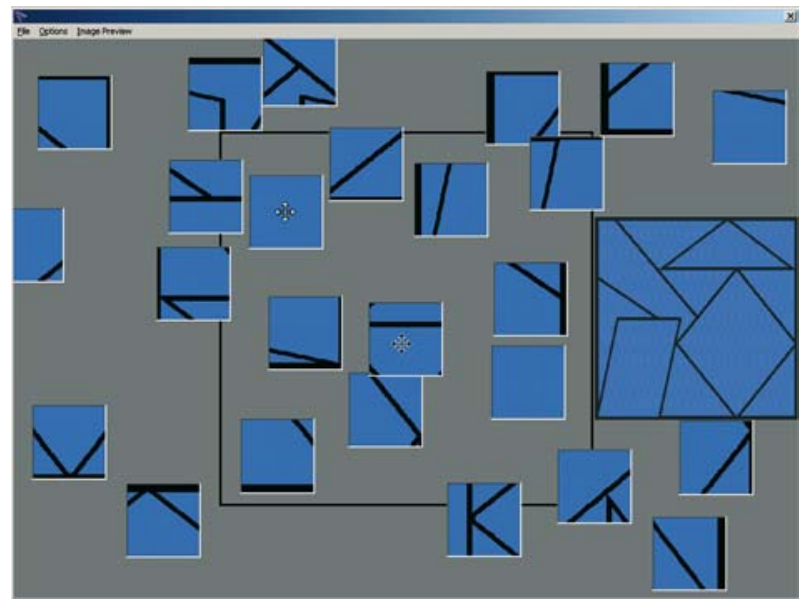

Figure 2. The geometric puzzle (electronic version).

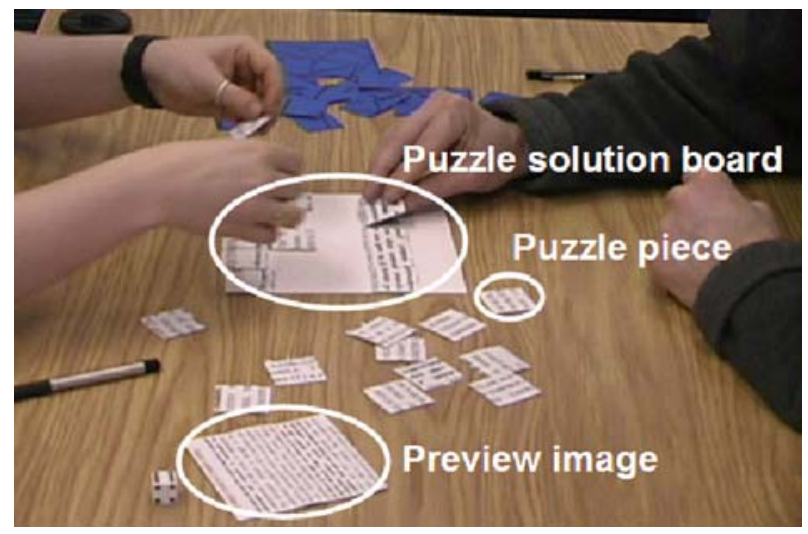

Figure 3. The text-based puzzle (paper-based version).

electronic puzzle, thereby allowing two users to simultaneously interact in the shared workspace. In allowing simultaneous interaction, design decisions regarding piece ownership were made so as to reduce conflict over puzzle pieces. For instance, when an individual had "picked up" a piece, another user was not able to manipulate that piece until the individual had released it. Simultaneous picking up of pieces was not allowed. These decisions mirror what typically happens in real-world puzzle completion. The paper-based puzzle for the nonelectronic tabletop condition was identical to the electronic puzzle in terms of the image used for each puzzle completed, and the size, shape and number of puzzle pieces (Figure 3). However, rather than using a black square border as the boundary for the completed puzzle, participants were provided with a square piece of cardboard on which to assemble the puzzle pieces. A preview 
image identical in size and shape to the electronic version was provided to participants as well.

\subsection{PARTICIPANTS}

Fourteen paid participants were recruited from the university population ( 5 females and 9 males). Seven observational sessions were conducted in total, with participants performing the study in pairs. The individuals and pairs chosen were quite distinct in many respects. Participants came from various academic departments, including computer science, environmental design, education, and management. There were frequent and infrequent computer users, as well as puzzle experts and puzzle novices. Some had experience with single display groupware while others had not. This purposed variation in the subject population was expected to provide a wide range of interactions during the puzzle exercises, which was hoped would contribute to a richer understanding of tabletop collaboration.

\subsection{EXPERIMENTAL DESIGN AND PROCEDURE}

In the traditional and digital table conditions, participants sat facing each other across the tabletop workspace. In the upright display condition, the participants sat side-by-side facing the computer monitor. Each participant was provided with a mouse to control their own on-screen cursor for the digital conditions. For the non-electronic tabletop condition, participants were free to position all puzzle-related items anywhere on the table. Each pair of subjects participated in the three collaborative conditions, completing an oriented and non-oriented puzzle for each condition. In general, pairs took less than 10 min to correctly assemble each puzzle.

Before the first experimental condition, participants filled out a consent form followed by a pre-session questionnaire. Then, the first of the three conditions (traditional table, electronic table, or upright display) was randomly chosen with the use of a die. The order in which participants completed the puzzles for this condition was also randomly selected, as were their seating positions. Participants were encouraged to employ the "think aloud" approach during task completion, which involved speaking out loud as often as possible to indicate what they were thinking. A video camera with a microphone was used to record the participants' actions and dialogue in each workspace setting for five of the seven sessions. After the two puzzles were completed in the first condition, the participants filled out a post-condition questionnaire providing comments on the activity. The above process was repeated for the remaining two conditions. After the third and final condition was completed, the users filled out a post-experiment questionnaire involving comparisons between the different conditions. The participants were then 
debriefed and thanked for their time. Each session took approximately one hour to complete.

\subsection{DATA ANALYSES}

Data were gathered from several sources during the study, including field notes, questionnaires and audio and video recordings of five of the seven sessions. In order to determine the interesting issues relating to tabletop interaction and how they differ from interacting with a vertical display, preliminary analyses were performed on the data. As a result of these preliminary analyses, it became apparent that orientation was a significant issue for tabletop collaboration, appearing to be more complex than previously identified. We saw almost constant use of orientation and its adjustment during collaboration over the traditional table. Consequently, an in-depth qualitative analysis of the oriented puzzle (i.e., text-based) in the traditional tabletop condition was performed. This condition allowed for examination of the specific ways in which orientation impacts traditional tabletop collaboration.

To help us understand this data, we developed a coding scheme containing orientation-related categories. We then applied this coding scheme to catalogue events captured by the video (and audio) data. Section 5 describes the coding scheme, how we used it to analyze the videotapes, and our numeric results.

\section{Video analysis of tabletop collaboration}

The video data consisted of approximately 50 minutes of five pairs of participants constructing puzzles with strongly oriented content (i.e., a passage of text). While participants also constructed puzzles containing non-oriented content (i.e., the geometric puzzles), we narrowed our analyses to only those sessions involving oriented content. This made sense given that our purpose was to understand the effects of orientation on collaboration, and it was made possible by the fact that the video data allowed the top and bottom of the oriented puzzle pieces to be visually distinguished.

\subsection{CODING SCHEME DEVELOPMENT}

From the preliminary analysis, our initial impressions were that people seemed to use orientation fluidly, without apparent conscious thought or planning as part of their non-verbal communication patterns. Also, the orientation of puzzle pieces that were not in active use at a given moment seemed to "speak" to the participants about whether or not those pieces were available for use. 
Table I. Behavioural instances used for the coding scheme

\begin{tabular}{|c|c|c|c|}
\hline Rotation & $\begin{array}{l}\text { Picking up/Putting } \\
\text { down }\end{array}$ & Comments/Gestures & Interactions \\
\hline $\begin{array}{l}\text { Rotation to establish } \\
\text { group orientation } \\
\text { Rotation of entire } \\
\text { puzzle } \\
\text { Rotation of } \\
\text { entire preview image } \\
\text { Rotation for self } \\
\text { Rotation for other } \\
\text { Rotation to } \\
\text { compromised angle } \\
\text { Rotation for piece } \\
\text { insertion } \\
\text { Rotation to } \\
\text { primary group } \\
\text { orientation } \\
\text { Rotation } \\
\text { to secondary group } \\
\text { orientation } \\
\text { Sharing } \\
\text { pieces in giver's } \\
\text { orientation } \\
\text { Sharing } \\
\text { pieces in receiver's } \\
\text { orientation } \\
\text { Rotation of groups } \\
\text { (i.e., } 2+ \\
\text { puzzle pieces) } \\
\text { Rotation within } \\
\text { personal space by self } \\
\text { Rotation within } \\
\text { personal space by } \\
\text { other } \\
\text { Rotation } \\
\text { within public space }\end{array}$ & $\begin{array}{l}\text { Picking up pieces } \\
\text { oriented for self } \\
\text { Picking up pieces } \\
\text { oriented for other } \\
\text { Picking up pieces at } \\
\text { compromised angle } \\
\text { Picking up pieces in } \\
\text { primary group } \\
\text { orientation } \\
\text { Picking } \\
\text { up pieces in } \\
\text { secondary group } \\
\text { orientation } \\
\text { Putting } \\
\text { down pieces oriented } \\
\text { for self } \\
\text { Putting down } \\
\text { pieces oriented for } \\
\text { other } \\
\text { Putting down } \\
\text { pieces at } \\
\text { compromised angle } \\
\text { Putting down pieces } \\
\text { in primary group } \\
\text { orientation } \\
\text { Putting } \\
\text { down pieces in } \\
\text { secondary group } \\
\text { orientation } \\
\text { Using } \\
\text { other person's } \\
\text { alignment (for } \\
\text { adding pieces, } \\
\text { discussing, etc.) } \\
\text { Using own alignment } \\
\text { (for adding pieces, } \\
\text { discussing, etc.) }\end{array}$ & $\begin{array}{l}\text { Comment relating to } \\
\text { establishing orientation } \\
\text { Comment relating to } \\
\text { established orientation } \\
\text { Comment suggesting } \\
\text { predefined orientation } \\
\text { Comment relating to } \\
\text { effect of orientation } \\
\text { Rotation involving } \\
\text { directed comment } \\
\text { Rotation not involving a } \\
\text { directed comment } \\
\text { Gesturing to ask for } \\
\text { rotation of piece } \\
\text { Gesturing to indicate } \\
\text { possibility of rotation } \\
\text { Gesturing involving } \\
\text { direct/close contact to } \\
\text { object of interest } \\
\text { Gesturing involving } \\
\text { distanced reference to } \\
\text { object of interest } \\
\text { Miscellaneous gesturing } \\
\text { related to orientation } \\
\text { Comment in response to } \\
\text { gesture } \\
\text { Comment in } \\
\text { response to head tilt } \\
\text { Comment in response to } \\
\text { standing up }\end{array}$ & $\begin{array}{l}\text { Orthogonal } \\
\text { rotation } \\
\text { Non-orthogo- } \\
\text { nal rotation } \\
\text { Temporary } \\
\text { rotation } \\
\text { Non-tempor- } \\
\text { ary } \\
\text { rotation } \\
\text { Rotation on } \\
\text { table } \\
\text { Rotation in } \\
\text { hands }\end{array}$ \\
\hline
\end{tabular}


From these impressions, we formed the following concrete questions as the basis for the coding scheme, as illustrated by the headings in Table I.

- Question \#1: How do people use rotations? For instance, do people use rotation to orient objects to their own best view?

- Question \#2: How does orientation affect people when they pick up and put down objects? How did people leave objects they were temporarily not going to use? Did an object's orientation influence the participant's subsequent behavior?

- Question \#3: Are comments and gestures important to people's understanding of orientation? Was use of orientation accompanied with words and/or gestures to clarify a person's intentions?

- Question \#4: What kinds of interactions were used when rotating objects? Was there any indication of how changes in orientation were performed that might be useful when designing collaborative tabletop software?

Based on these questions, we revisited the video data to look for specific classes of actions that seemed to fit within these questions (as fully listed in Table I). For example, in column 1 we list 15 actions related to how people used rotation; in column 2 we list 12 actions related to how people picked up and put down objects, and so on. Each of these actions then became a coding category for the video analysis.

\subsection{TRANSCRIPTION}

Orientation-related actions, such as rotating or picking up and putting down puzzle pieces, are very short and rapid. Several may happen virtually simultaneously, either because more than one person is active in the workspace at a given moment, or because the actions happen in quick succession. To help codify and analyze these series of actions, we created an application to assist in transcription that provided a high-resolution video image which could be controlled via the keyboard. During transcription, each action from Table I was given an abbreviated code; for example, rotating an object for

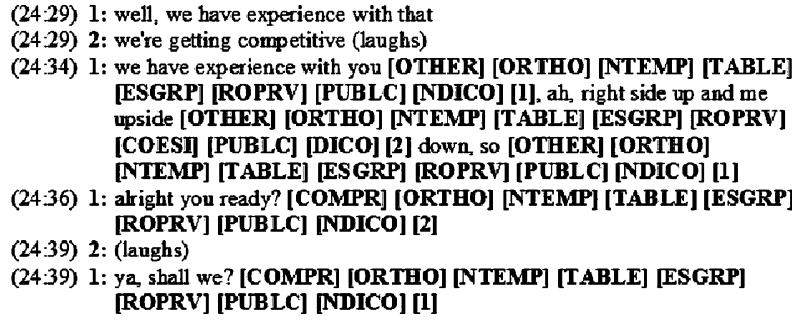

Figure 4. Transcript segment showing interaction between two participants. Notice how tightly the codes are spaced. 
one's self was simply coded as [SELF]. Figure 4 gives an example of a transcription segment. The numbers on the left represent the elapsed time, and " 1 " and " 2 " refer to dialogue for participant 1 and 2, respectively. The items in square brackets (e.g., [OTHER]) each represent a code, with the exception of [1] and [2] which indicate the person to which a code or group of codes apply. Sequences of codes up to a [1] or [2] represent one specific action or item of interest.

Three of the five videotapes were transcribed and coded in this manner. After video transcription was completed, the resulting transcripts were analyzed in three primary ways. The first involved developing frequency counts of codes. The point of this analysis was not to make statistically significant claims about the data, but rather to determine the relative occurrence of actions. The second involved identifying the co-occurrence of particular codes, to see if and how they were related. The third approach involved highlevel inspection of all five videotaped sessions and completed transcripts in an effort to capture interesting examples that were not previously identified.

\subsection{NUMERICAL RESULTS}

The completed transcripts contained nearly 1000 codes representing slightly more than 250 identifiable actions. Many actions have more than one code. For instance, a given rotation might be: orthogonal (oriented at a $90^{\circ}$ angle to the table or the workspace), rotated for one's self, non-temporary and done on the table. Of the identifiable actions

- fourty percent are rotations of one type or another,

Table II. Numerical results for Question \#1: Rotations

\begin{tabular}{lc}
\hline Action & Relative percentage \\
\hline Rotation for self & $32.7(32 / 98)$ \\
Rotation for other & $34.7(34 / 98)$ \\
Rotation for both & $32.7(32 / 98)$ \\
Rotation within personal space by self & $23.5(23 / 98)$ \\
Rotation within personal space by other & $0.0(0 / 98)$ \\
Rotation within public space & $35.7(35 / 98)$ \\
Rotations not categorized in this manner (for instance, rotations & $40.8(40 / 98)$ \\
performed in one's hands) & \\
Rotation according to primary group orientation & $66.3(65 / 98)$ \\
Rotation according to secondary group orientation & $25.5(25 / 98)$ \\
Rotation according to neither group orientation & $8.2(8 / 98)$ \\
\hline
\end{tabular}


- fifty-six percent are actions of picking up and putting down pieces (note that pieces that were successfully placed in their correct location in the puzzle were not counted in this), and

- four percent are accompanying comments and gestures.

Numerical results of the video analysis are presented below grouped according to the questions introduced in Section 5.1.

Question \#1: Rotations. Table II presents results concerning how rotations of puzzle pieces were performed. The first three rows show that rotations were fairly evenly split between rotating for one's self, rotating for the other person, and rotating to allow both individuals to view a piece at a compromised angle. The second four rows show that a person either performed rotations in the space directly in front of them (their personal space) or in the space surrounding the puzzle (public space), but never in the space directly in front of the other person. The last three rows show the split between rotating objects according to the primary vs. secondary group orientation (with primary referring to the orientation of the square on which the puzzle was assembled and secondary referring to the orientation of the preview image - these group orientations are discussed in more detail in Section 6.2). In terms of the remaining behavioral instances related to Question \#1 given in Table I, there were very few instances of two people sharing one puzzle piece (only 3 ), but when they did it was always in the receiver's orientation. Also, there were no instances of rotating groups of puzzle pieces simultaneously. It should be noted that the relative percentage for each cluster of rows in Table II adds to $100 \%$, as each cluster examines the data from a differing and separate perspective.

Table III. Numerical results for Question \#2: Picking up and putting down pieces

\begin{tabular}{ll}
\hline Action & Relative percentage \\
\hline Picking up pieces oriented for self & $33.6(39 / 116)$ \\
Picking up pieces oriented for other & $13.8(16 / 116)$ \\
Picking up pieces at compromised orientation & $52.6(61 / 116)$ \\
Putting down pieces oriented for self & $24.3(9 / 37)$ \\
Putting down pieces oriented for other & $27.0(10 / 37)$ \\
Putting down pieces at compromised orientation & $48.6(18 / 37)$ \\
& $57.8(67 / 116)$ \\
Picking up pieces in primary group orientation & $28.4(33 / 116)$ \\
Picking up pieces in secondary group orientation & $13.8(16 / 116)$ \\
Picking up pieces in neither group orientation & $64.9(24 / 37)$ \\
Putting down pieces in primary group orientation & $32.4(12 / 37)$ \\
Putting down pieces in secondary group orientation & $2.7(1 / 37)$ \\
Putting down pieces in neither group orientation & \\
\hline
\end{tabular}


Table IV. Numerical results for Question \#3: Combining orientation with comments or gestures

\begin{tabular}{ll}
\hline Action & Relative percentage \\
\hline Rotations involving a directed comment & $15.3(15 / 98)$ \\
Rotations not involving a directed comment & $84.7(83 / 98)$ \\
\hline
\end{tabular}

Question \#2: Picking up and putting down pieces. Table III presents results related to the orientation of puzzle pieces as they were picked up and put down by participants. The first three rows show that people were more likely to pick up objects that were either oriented for themselves or at a compromised angle. The second group of three rows in Table III shows the split between putting pieces down at an angle that favored one's self, one's collaborator, or both individuals. The last six rows show that it was more likely that a person picked up and put down pieces that were oriented according to the primary group orientation.

Question \#3: Combining orientation with comments or gestures. Table IV shows that very rarely did either a comment or a gesture accompany an action that involved orientation. In regards to the behavioral instances related to Question \#3 given in Table I, there were only two comments made that referred to the established group orientation, one comment about orientation in response to a gesture and no comments about either predefined orientations or the effect of orientation. Only two separate gestures were made that related to orientation, and no gestures were made associated with rotating a puzzle piece.

Question \#4: Interactions. Table V shows that there were slightly more orthogonal than non-orthogonal rotations and relatively few temporary rotations. Slightly more rotations were performed on the table than when pieces were being held in a person's hands.

Table V. Numerical results for Question \#4: Interactions

\begin{tabular}{lc}
\hline Action & Relative percentage \\
\hline Orthogonal rotations & $56.1(55 / 98)$ \\
Non-orthogonal rotations & $43.9(43 / 98)$ \\
Temporary rotations & $7.1(7 / 98)$ \\
Non-temporary rotations & $92.9(91 / 98)$ \\
Rotations on table & $55.1(54 / 98)$ \\
Rotations in hand & $44.9(44 / 98)$ \\
\hline
\end{tabular}


Table VI. Three roles of orientation

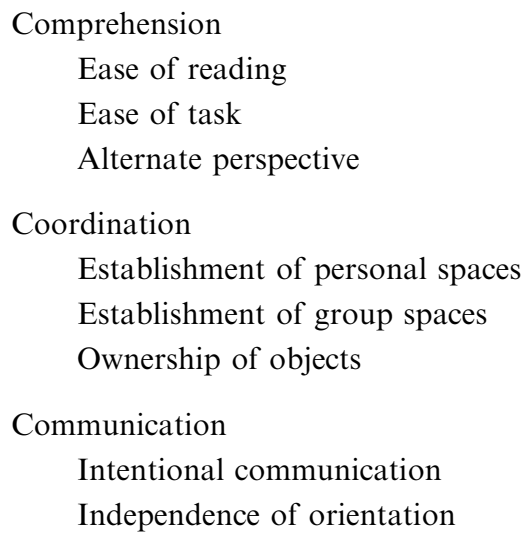

\section{The three roles of orientation}

Through critical analysis of the previous literature, results of our formative studies, and the in-depth analysis of tabletop collaboration reported in Section 5, we have identified three key roles of orientation that impact collaboration and have implications for the design of tabletop interfaces: comprehension, coordination, and communication. We can break down each of these key roles of orientation into several more specific roles (Table VI). The next three sections will describe each of these orientation roles in turn and provide supporting examples from our data sources.

\subsection{COMPREHENSION}

The most obvious use of orientation is comprehension. In practice, people often rotate tabletop items to help themselves read (or draw or write on) the item. We will also see in Section 6.3 that people also rotate items to help others $\mathrm{read} / \mathrm{draw} / \mathrm{write}$ on the item. The role of orienting items for comprehension is fairly basic, as we know that it is often difficult to interpret something that includes text or symbols unless it is oriented correctly. Yet while it may seem that there is only one 'correct' or 'best' orientation for an individual viewing an item, people also rotate items to different angles to help them understand or interact with the content. These nuances are discussed below.

\subsubsection{Ease of Reading}

People often orient objects so that they are most readable for themselves. Usually this involves turning the object so that it is the 'right way up' so that words and symbols are easily read and interpreted. This role of orientation is recognized by most tabletop technology designers, who often try to orient an 


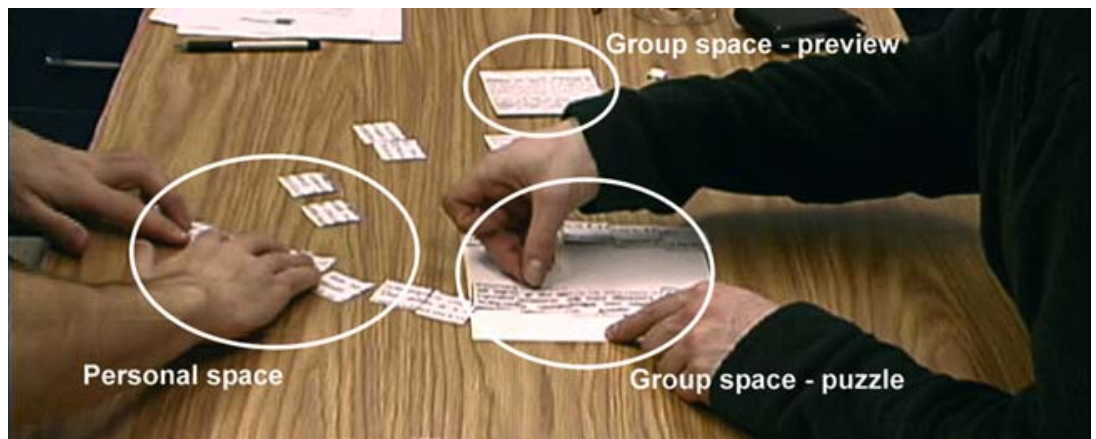

Figure 5. An example of personal and group spaces.

object 'right way up' for the user who is either currently accessing it or is closest to it (Rekimoto and Saitoh, 1999; Shen et al., 2001, 2002; Vernier et al., 2002).

However, our data revealed that the 'right way up' does not necessarily mean that items face the edge of the table where a person is seated, as is often done in both person-based and environment-based automatic orientation strategies (Section 2). Instead, the items could be oriented tangential to how the person is looking at the item, i.e., how they move their head and eye gaze towards the item. Figure 5 gives an example, where we see an image extracted from a video of two people collaborating on a puzzle. The person on the left is sitting somewhat sideways to the table, and consequently, he has aligned his pieces towards himself (and the best viewing angle), rather than to the table's edge.

\subsubsection{Ease of Task}

People may also rotate items on the table to a position that provides the best angle for completing a given task. For example, Fitzmaurice et al. (1999) reports on various studies of artists that describe: how they operate within an articulation comfort range as they draw; how they rotate artwork so their hand does not obscure the key area of the drawing; and how they are careful to position the drawing so as not to smear or damage sensitive parts of the drawing, for example, those with wet paint. These angles may, of course, differ from the reading orientation. For example, we know that people may use a tangential orientation for reading, but a different 'slanted' orientation when writing for comfort's sake (Guiard, 1987).

\subsubsection{Alternative Perspective}

People also rotate items in various ways to help them understand its content. These rotations provide people with alternative perspectives of the item, especially if the item has multiple orientations or is not strongly oriented. Obtaining alternate perspectives is accomplished by rotating items on the 
table, or by a person moving to a different location around the table. This happens, for example, when a chess player walks around a chess table.

\subsection{COORDINATION}

Tang (1991) observed that orientation appears to play a mediating role in the coordination of actions between individuals in a collaborative setting. To understand how this happens, we observed and analyzed the subtleties of how people use orientation as a coordinative act. In particular, we saw that orientation of items is used by people to establish different categories of personal and group spaces on a table, and to communicate ownership or accessibility of these items.

\subsubsection{Establishment of Spaces}

A horizontal display serves as both a shared space for doing group work, and a personal space for doing individual work. Yet there is usually no explicit demarcation on the surface itself as to what comprises the personal vs. group space. Consequently, people divide the space through other means, for example, explicit verbal demarcation (this is mine, this is yours), or implicit demarcation in terms of where people place objects and how they are oriented. In practice, orientation proves an important cue to others in how personal and group spaces are created and communicated and how subsequent actions over the surface occur.

Establishment of a personal space through orientation. One way we saw that people established their personal space was to orient objects in an area of the tabletop so that they were 'just right' for their own use (Section 6.1). This in turn creates a space less usable by others and where others tend not to perform actions. Typically, personal objects are kept close to the person they belong to (see also Tang, 1991) and are oriented appropriately for him or her. This makes objects easier for that person to see, read and use for one's task, and harder for others to see, read and use.

In our puzzle study, most people created their own personal space by orienting puzzle pieces appropriately in the area directly in front of them. Figure 5 shows this for the participant seated on the left, where he has clearly organized his collection of puzzle pieces both by proximity and by tangential orientation; this creates a spatial region that suggests these items are for his own use in the current task. Note that items around this personal zone are oriented differently from other items in the workspace.

Establishment of a group space through orientation. A group space is one or more spaces in which more than one person feels free to work. While this may occur as either an implicit or explicit group decision process, it usually takes place with great ease. Unlike the personal space where both 
proximity and orientation favor a particular person, a group space may compromise some or all participants because the space is typically located somewhat centrally on the table, and because items may be 'upside down' for some participants. Yet people willingly and gracefully accept this compromise.

In the puzzle study, we saw that all groups easily and quickly established a group orientation at the beginning of the puzzle task. No one said they minded what their relationship would be to the established group orientation. In fact, some people were proud of their skill at working with items that were 'upside down' for them.

We also saw that multiple group spaces can co-exist, and each can have a different orientation. In particular, we saw some people establish two group orientations for the puzzle task. The first was the orientation of how the puzzle would be assembled (the primary task), and the second was the orientation of the preview image (Figure 5). These orientations were usually done quickly, where only a handful of physical actions and interpersonal negotiations established the group orientation.

Establishing group orientation proved to be a very social act. In every case, the person responsible for establishing the group orientation attempted to favor the other person by aligning the puzzle 'right way up' for their collaborator. Figure 6 gives an almost comical example of the lengthiest negotiation we saw for establishing the orientation of the preview image. In frame A, Participant 1 (on the right) initially suggests an orientation that makes the image 'right way up' for Participant 2 (the bottom of the image is indicated by a thick black line). In frame B, Participant 2 counters by rotating it so that it is 'right way up' for Participant 1. This back-and-forth continues, until Participant 1 suggests a compromise between the two orientations in frame D, which is then flipped by Participant 2 and accepted by both in the final frame.
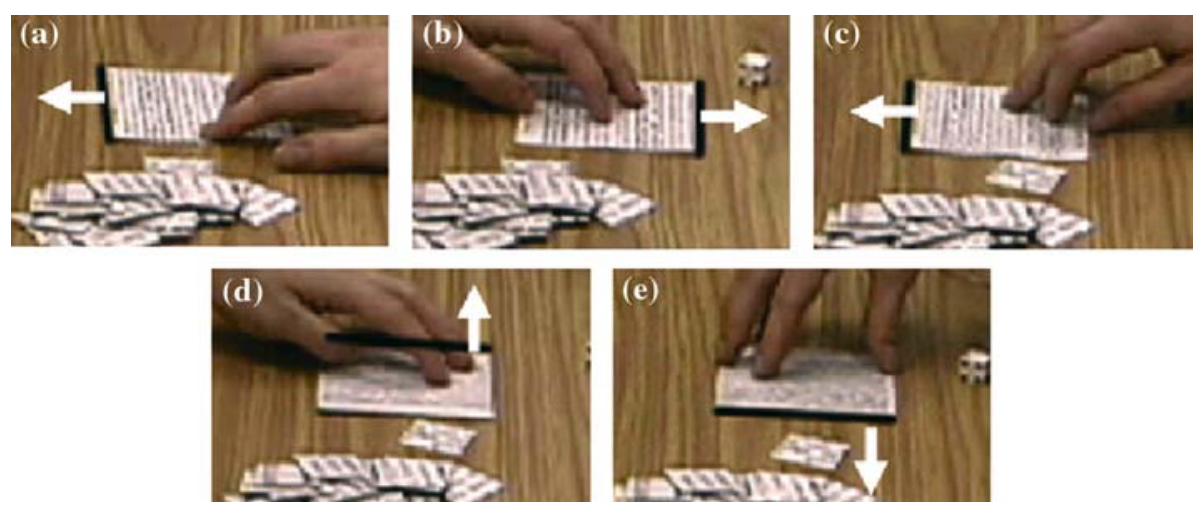

Figure 6. Negotiating group orientation. 
Once established, the group orientation had a significant effect on the completion of the puzzle. For example, approximately two-thirds of all rotations were to turn pieces to the same orientation as the puzzle; this makes sense, as the primary goal of participants was to fit the pieces into the puzzle. However, other rotations were done with respect to the preview image, i.e., so a person could get a sense of how and where their piece would eventually fit into the completed puzzle.

Finally, we should add that the distinction between personal and group orientation is not necessarily permanent; one group altered the preview image from a group orientation to a more personal orientation. The image then fluctuated between being both a group and personal resource.

\subsubsection{Ownership of Objects}

Groupware systems often have a strong notion of ownership, where the system dictates access control and restricts who can do what. In real life, however, ownership and control is often a socially mediated process, determined by implicit subtleties such as proximity, the history of who has used an object, and so on. Because ownership can change rapidly during collaboration, the heavyweight notions of access control often found in groupware may be inappropriate for co-located collaborative settings.

Orientation also serves to mediate the ownership of tabletop objects. In particular, our data revealed two ways that orientation dictated who 'owned' a particular object, and how others were willing to respect that ownership.

- Orientation for picking up/using objects. People are much more likely to pick up and use objects that are oriented towards themselves or at a compromised angle.

- Placing oriented objects for availability. The way people place an object suggests personal ownership/access if the object is oriented towards themselves, and shared ownership/access if it is oriented towards others or at a compromised angle.

Our video analysis suggests that the control and ownership of items within a personal space was rarely in dispute. In particular, we counted how people picked up and put down puzzle pieces with respect to their orientation. First, in all cases, only the 'creator' of the personal space (similar to the one illustrated in Figure 5) would rotate or access the pieces within that space. Second, out of 116 observed actions, people picked up pieces facing them about $33 \%$ of the time, pieces oriented at a compromised angle about $53 \%$ of the time, and pieces oriented towards others about $14 \%$ of the time. That is, a person is much more likely to pick up objects that are set according to a compromised orientation or that are oriented for themselves. A person is much less likely to pick up a piece oriented directly towards someone else. 
Our data also suggest that people place pieces on the table at these angles as an indication of ownership and/or how the item should be shared. For example, if people did not think communally, we would expect them to predominantly place objects in an orientation that was suitable for them. Yet this happened only about $24 \%$ of the time. We saw that people were very likely to place pieces at an angle that was either compromised (49\%) or directed towards the other person (27\%). We also saw that if people picked up pieces oriented to the group orientation, they usually put them down in the same orientation. This indicates that people replace shareable objects in a way that tells others that the object is still communally owned and publicly accessible.

\subsection{COMMUNICATION}

Orientation also plays a mediating role in communication between individuals in a collaborative setting. In particular, orientation is used as an intentional communicative act and is independent from other patterns of communication.

\subsubsection{Intentional Communication}

Intentional communication is prevalent between people as an explicit mechanism for gathering and exchanging information (Clark, 1996). We usually see this in face-to-face discussions via people's intentional verbal exchanges and by the explicit hand and body gestures that accompany and accentuate talk (Pinelle et al., 2004). This also occurs over workspaces via talk and deictic gestures (Tang, 1991). Our observations of tabletop collaboration suggest that how people orient objects when working over tabletops also serves a role in intentional communication.

- Orienting an object to oneself signals no intentional communication; that is, the person is doing their own personal work.

- Orienting an object to another person signals that the object, the person's talk, and any accompanying gestures are being directed towards a particular person for communicative purposes. If the item is oriented directly towards the other person, this typically establishes an audience or indicates relinquishment of turn. If the item is oriented at some compromised angle, this almost invariably initiates a response in the form of discussion and a period of close collaboration.

- Orienting an object to the group is similar, except that the objects and any accompanying talk and gestures are now being directed towards the group (or sub-group).

Our observations confirm the role of orientation in intentional communication. People intentionally rotated objects as part of their communicative acts 
after they had established their personal and group spaces. About one-third of all rotations were to oneself, signaling no communication. This was recognized by others in that they left those objects alone and any gestures or adjustments made to them were ignored or not noticed.

About another third of people's rotations involved directly orienting an item to another person's perspective. This alignment of an object so that it was 'right way up' for the other person proved to be one way of directing communication towards them. We also noticed that when people shared an item with another person by passing it to them, the giver always oriented the item according to the orientation of the person receiving the piece.

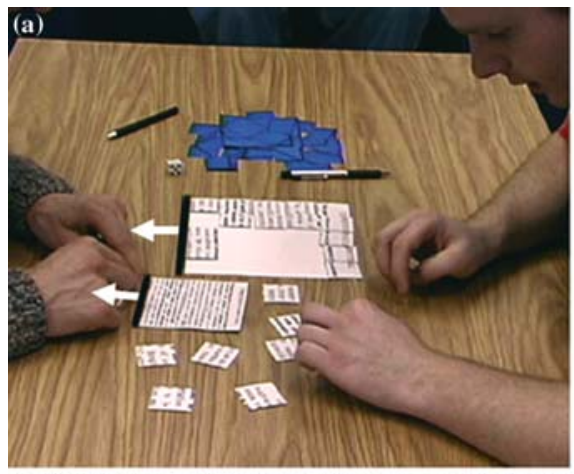

Participant 1 (left) reads the preview image. Participant 2 (right) looks at puzzle pieces.

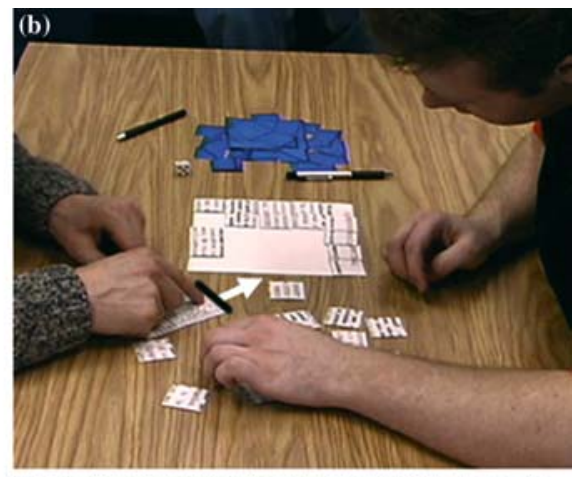

Participant 1 rotates the preview image to an angle that is very compromised for him and slightly compromised for Participant 2. Participant 2 immediately responds by tilting his head.

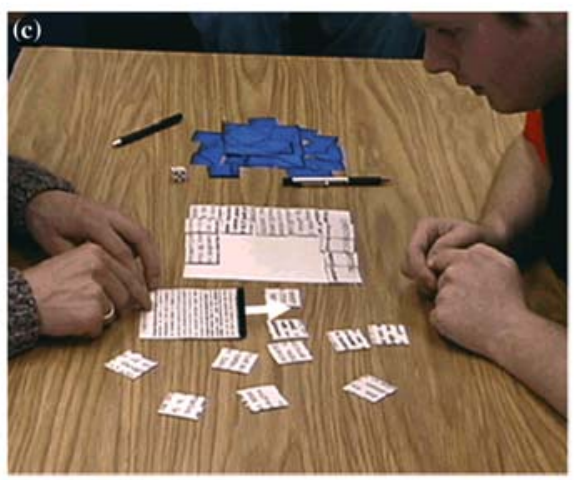

Collaboration is established and the two participants proceed to work together. The image is now completely oriented towards Participant 2.

Figure 7. Two people establishing collaboration using object orientation. 
The remaining one-third of all acts involved rotating items to a compromised angle, i.e., an orientation that made the item visible to both people but which was not aligned well for any one person (for instance, as in the group workspace in Figure 5). This clearly signaled to others that the item was to be a focus of discussion or engagement for both parties. This partial rotation is of particular interest to the collaborative process. When a rotation is made to an orientation that is compromised but possible for both people to view, it appears to be a very compelling communicative gesture. In our study, this action always initiated discussion and seemed to be a well-understood method for starting immediate collaboration. As well, we observed a reciprocal head tilt by the receiving person to indicate a willingness to read at a compromised angle. Both of these actions seemed quite compelling. Moreover, we have noticed this action-response pairing in a variety of situations during our day-to-day interactions.

The sequence of images in Figure 7 illustrates this process. In frame A, both participants are doing their own work and Participant 1 (on the left) is looking at the preview image, which is 'right way up' for him. In frame B, Participant 1 rotates the preview image towards Participant 2. Participant 2 notices and tilts his head in response. In frame $\mathrm{C}$, the collaboration is well in hand: Participant 1 is reading, though the text is now upside down for him, and Participant 2 is assembling pieces that match.

\subsubsection{Independence of Orientation}

Non-verbal conversational acts are often tied to other intentional communication as a way to explain or clarify that person's intentions or to remove ambiguity. For example, talk and gestures often work together, e.g., as in deictic references. Orientation, however, proves to be an understandable stand-alone act that does not require additional communication in the following cases.

- Orientation independence as one repositions an object. As people pick up, use, and reorient objects, they rarely comment or add gestures to explain such rotation actions.

- Orientation independence of objects already positioned. For an object already placed on the table, its orientation informs others as to whether or not it is available. No further requests for information are needed.

Our analysis of orientation during tabletop use showed that people rarely accompanied a rotational act with a directed comment or additional gesture to another person (only about $15 \%$ of the time) - and when they did, more than half of these were comments made when the group initially established the group orientation. Thus, the vast majority of rotations $(85 \%)$ did not involve any directed comments whatsoever. Accompanying gestures were rarely seen. Hence, rotation is a relatively "lightweight" communication 
activity that people do naturally, quickly, intuitively, and without explicit consideration. Similarly, people rarely asked for explanations or commented on items already positioned on the table. That is, the meaning of the orientation was self-explanatory.

Thus, people appear to be aware of the meaning of orientation changes both as they happen and afterwards. The orientation act and object position are usually sufficient for communicating to others about who 'owns' what, what one is currently using, and what one is finished with.

Because of this independence of orientation, the alignment of items left as a result of work on the tabletop continues to inform the participants. This effect can be described as the residual communication of objects; that is, for an object already positioned on the table, its orientation informs others as to whether or not it is available. No further requests for information are needed.

\section{The mechanisms of orientation}

We have just discussed how orientation plays several important roles in tabletop collaboration. As will be discussed in Section 8, we believe that digital tabletops should be designed to facilitate how people make use of these various roles of orientation. Additionally, people should be provided with suitable interaction techniques for manipulating the orientation of objects on tabletop systems. Consequently, we articulate orientation mechanisms exhibited by collaborators during the course of our study to inform the development of such interaction techniques.

Analysis of our observational data revealed several basic mechanisms that contribute to the comprehensive, coordinative, and communicative roles of orientation. These include the angle or degree of rotation (orthogonality), the duration of rotation, and the location of rotation.

Orthogonality. As previously discussed, many current solutions to tabletop orientation position on-screen items orthogonally to the table edge, usually aligned with whichever edge the system believes the user is located. If users are located on different sides of the table, this positioning typically favors only one person at the table.

Our study analysis revealed that collaborators made both orthogonal and non-orthogonal rotations of objects during completion of the task. However, even with an orthogonally-biased puzzle application (i.e., one with square puzzle pieces, a square solution board and a square preview image), only slightly more than half the rotations resulted in square-alignment with an established orientation. The remaining rotations resulted in non-orthogonal alignment. This strongly indicates that tabletop software needs to handle both variant and orthogonal orientations. For example, once associated orientations have been established in personal and group spaces, it may be 
possible to provide automatic orientation as items move into these spaces. Yet since variant rotations occurred in all spaces, it should be easy to override automatic rotations to allow an object to be oriented to any desired angle.

Duration. Another question concerns the temporal nature of rotations. As previously mentioned, one of the more powerful communicative uses of orientation is when a person shows a collaborator an item held at a compromised angle. It is during the actual act of rotation that communication is typically initiated (Figure 7). While the rotation used to initiate the communication is often temporary, we found a decided tendency to leave items in their last position when the discussion ended. Rather than just being untidy, the orientation of the items continued to inform people about which items were available for use (see Section 6.3.2). As a result, the vast majority of rotations were non-temporary $(93 \%)$. Hence, the ability to effortlessly rotate objects while communicating, as well as the ability to stop that rotation at any point, may be important to support in a tabletop interface.

Locations of interaction. For the tabletop interface designer, it is important to know if the various uses of orientation were parts of interaction that occurred directly on the tabletop surface or in the space above the table. Orientation-related actions taking place on the surface of the table are much more accessible for supporting in software. Actions taking place above the table require additional interactional support, such as including support for gestures or tangible interface components. In the study, about half of all rotations were performed on the tabletop surface. As well, it should be noted that these rotations were used for all three purposes of orientation: comprehension, communication and coordination. Hence, it seems likely that tabletop software can be designed to leverage people's familiarity with twodimensional rotation. However, the fact that almost half of all rotations took place above the table's surface does raise design questions regarding the importance of non-traditional input for tabletop displays.

\section{Implications for design}

Generations of people have gathered around tables in boardrooms, meeting rooms, and cafés for a variety of collaborative activities. These activities range from games and leisure activities to design and planning activities. Consequently, most people will have preconceptions about the types of activities possible on a digital tabletop display and the collaborative benefits of using a tabletop workspace. Therefore, in order to preserve the benefits of using a tabletop environment for collaboration, the comprehensive, coordinative, and communicative roles of orientation must be supported in the information layout strategies and rotation interaction techniques of a tabletop interface. 
Our observational data has several implications for the design of such layout and interaction techniques as outlined below.

Free rotation must be supported. The observational data showed many instances of both full and partial rotations, as well as orthogonal and nonorthogonal rotations. A system that provides techniques to rotate objects to any angle would allow people to perform all such rotations. It would also allow people to place objects correctly in already established oriented spaces, such as group or personal spaces.

Rotation techniques must be lightweight. Participants in our study performed numerous and rapid object rotations during the tabletop collaboration. These would be inhibited if interaction techniques were heavyweight, such as those typically seen in conventional object-drawing packages. Instead, we need lightweight interaction techniques that place minimal overhead on performing a rotation operation. Such techniques would minimize interference with the comprehensive, coordinative, and communicative roles for which the rotations are being used. Lightweight interaction techniques would also allow users to establish or change the orientation associated with personal and group spaces quickly.

Orientation of user-positioned items must be maintained. Our observational data suggest that whether an item is currently available for use depends both on its current location (e.g., the oriented space in which it is located) and its current orientation (e.g., an individual may have rotated an item towards a collaborator). A tabletop system should avoid reorienting such items without the user's (implicit or explicit) permission to avoid inadvertently changing the information being communicated about an item's availability. Such system action could interfere with the coordination of the collaborative activities.

Rotation actions must have clear feedthrough. In order to preserve the nonverbal communicative role of orientation, it must be obvious to others when a user is performing a rotation action. Otherwise, the action may be missed. Yet, many groupware systems have historically removed or stylized finegrained actions associated with object movement for the sake of system performance. Other systems provide single-step rotations (e.g., through a menu selection) that could easily be missed by other participants. To provide the long-term communicative effects of orientation (e.g., conveying the ownership or availability of an item), the system should also clearly show an item's orientation both during and after the rotation action.

Automatic support for rotation and orientation must be handled carefully and allow easy user override. One way to go beyond conventional tabletops is to support automatic rotation, ostensibly to minimize an individual's work. While our results clearly show that there are areas on the table that suggest an orientation, there are also many times where people choose variant orientations in these areas. Thus, any automated rotation performed by the system must be carefully designed to balance the comprehensive needs of 
individuals viewing tabletop items and the group coordination and communication needs essential to the collaborative process. Systems which perform automatic rotation should provide users with lightweight mechanisms to override these system actions so they can position items to meet their current needs.

Table VII. Comparison of existing systems based on the design criteria suggested by the implications

\begin{tabular}{lllllll}
\hline Design criteria & \multicolumn{2}{l}{ Tabletop systems } \\
\cline { 2 - 7 } & ConnecTable & InfoTable & $\begin{array}{l}\text { Café } \\
\text { Table }\end{array}$ & InteracTable & PDH & UbiTable \\
\hline Free rotation & $\checkmark$ & $\checkmark$ & $\times$ & $\checkmark$ & $\checkmark$ & $\checkmark$ \\
Lightweight & $\checkmark$ & $\checkmark$ & $\times$ & $\checkmark$ & $\checkmark$ & $\checkmark$ \\
Orientation & $\checkmark$ & $\checkmark$ & $\checkmark$ & $\checkmark$ & $\times$ & $\checkmark / \times$ \\
maintained & & $\checkmark$ & $\times$ & $\checkmark$ & & \\
Feedthrough & $\checkmark$ & $\times$ & $\times$ & $\times$ & $\times$ & $\times$ \\
Override & $\times$ & $\times$ & & & & \\
\hline
\end{tabular}

Table VIII. Comparison of existing systems based on the roles of orientation

\begin{tabular}{|c|c|c|c|c|c|c|}
\hline \multirow[t]{2}{*}{ Roles } & \multicolumn{6}{|c|}{ Tabletop systems } \\
\hline & ConnecTable & InfoTable & $\begin{array}{l}\text { Café } \\
\text { Table }\end{array}$ & InteracTable & $\mathrm{PDH}$ & UbiTable \\
\hline \multicolumn{7}{|l|}{ Comprehension } \\
\hline Reading & $\checkmark$ & $\checkmark$ & $\checkmark$ & $\checkmark$ & $\checkmark$ & $\checkmark$ \\
\hline Task & $?$ & $?$ & $\checkmark$ & $?$ & $\checkmark$ & $\checkmark$ \\
\hline Perspective & $\checkmark$ & $\checkmark$ & $x$ & $\checkmark$ & $\checkmark$ & $\checkmark$ \\
\hline \multicolumn{7}{|l|}{ Coordination } \\
\hline $\begin{array}{l}\text { Personal } \\
\text { spaces }\end{array}$ & $\checkmark$ & $\checkmark$ & $\times$ & $\checkmark$ & $x$ & $\checkmark$ \\
\hline Group spaces & $\times$ & $\checkmark$ & $\checkmark$ & $\checkmark$ & $\checkmark$ & $\checkmark$ \\
\hline Ownership & $\checkmark$ & $\checkmark$ & $x$ & $\checkmark$ & $x$ & $\sqrt[J]{ } / \times$ \\
\hline \multicolumn{7}{|l|}{ Communication } \\
\hline Intentional & $\times$ & $\checkmark$ & $x$ & $\checkmark$ & $\sqrt{ } / \times$ & $\checkmark / x$ \\
\hline Independence & $\checkmark$ & $\checkmark$ & $\checkmark$ & $\checkmark$ & $\times$ & $\sqrt{ } / \times$ \\
\hline
\end{tabular}




\section{Implications for assessment}

Understanding the roles orientation plays during tabletop collaboration provides a means to compare existing approaches to orientation. Current tabletop systems can be evaluated based on how well they support the various roles of orientation. The design implications presented in the previous section can be used to guide such evaluations. From these assessments, we can leverage the strengths of each approach to create more effective rotation and orientation solutions.

To illustrate how both the roles of orientation and their design implications can be used to evaluate existing digital tabletop systems, we present a brief comparison of six existing systems previously introduced in Section 2: the ConnecTable (Streitz et al., 2001; Tandler et al., 2001), the InfoTable (Rekimoto and Saitoh, 1999), the Café Table (de Bruijn and Spence, 2001; Stathis et al., 2002), the InteracTable (Streitz et al., 1999, 2001), the Personal Digital Historian (PDH) (Shen et al., 2001, 2002; Vernier et al., 2002), and the UbiTable (Shen et al., 2003). A summary of this comparison is presented in Tables VII and VIII. In these tables a checkmark $(\checkmark)$ indicates that, for the most part, a system addresses the roles of orientation or the design criteria. If it does not appear to sufficiently address the roles or design criteria, we indicate this with an ' $x$ '. In some cases, only certain rotation or orientation techniques provided by a system address the roles or design criteria; this is indicated by a hybrid symbol $(\boldsymbol{} / \times)$. Finally, in the case of the Ease of Task role for Table VIII, it was difficult to determine whether a system addressed this role if no real applications exist for the system (as discussed below); this case is indicated by a question mark (?).

To preface our evaluations, we acknowledge that most tabletop systems (e.g., ConnecTable, and InfoTable, InteracTable) are prototype interfaces that generally demonstrate simple operations on primitive tabletop items, such as images, sketches, and icons. In contrast, PDH, the UbiTable, and the Café Table offer more complex applications designed for specific purposes: story-sharing, document sharing, and information viewing and sharing, respectively. Hence, certain design decisions have been made in these systems to provide specific functionality, which sometimes limits their ability to flexibly address orientation.

Overall, the emphasis of these systems has been on orienting objects in an attempt to align them 'right way up' for individuals. While clearly attempting to address the comprehensive role of orientation, the coordinative and communicative roles of orientation have been less universally supported.

First, we examine how the six tabletop systems address the design criteria suggested by our design implications. Then, we discuss the interplay between these design criteria and the roles of orientation as it relates to developing tabletop systems which facilitate collaboration. 
Of the six systems, the Café Table is the only system that does not allow free rotation. The other five systems all provide relatively lightweight mechanisms for rotating tabletop objects to any desired degree of rotation. The InfoTable, InteracTable, and ConnecTable provide free rotation via circular gestures performed on tabletop items. PDH and the UbiTable provide a rotation handle on the bottom-left corner of workspace items for manual, free rotation, while also providing several lightweight rotation mechanisms aimed at comprehension and communication. In these systems, a global rotation mechanism is used to rotate the entire central workspace like a "Lazy-Susan," while a "passing" mechanism automatically reorients an object being moved across the central workspace towards the outer edge of the workspace. This allows an item moved directly in front of an individual to be oriented facing that person. Similarly, the InteracTable provides a lightweight "toss" gesture to quickly move an item from one side of the table to another.

The combination of a rotation gesture and the animated rotation of the item being acted upon in the InfoTable, ConnecTable, and InteracTable systems provides clear indications of feedthrough. Rotating an object in $\mathrm{PDH}$ or the UbiTable using either the rotation handle or the passing mechanism also provides clear feedthrough, since objects are acted upon directly and the rotation occurs in sync with the user's action. However, globally applied orientation changes that occur with the Lazy-Susan workspace rotation (provided in both the UbiTable and PDH systems) and the "Magnet" tool (provided in PDH) may not provide clear feedthrough. These global rotation actions indiscriminately rotate all tabletop items in the central workspace, which affects the orientation of user-positioned items on the table. The other systems, however, maintain the orientation of workspace objects until someone acts on them further.

None of the six systems provide adequate user override mechanisms for automated rotation and orientation strategies. Peripheral icons on the Café Table continuously scroll around the workspace, aligned by the system tangentially to the curved table edge. The user has no way of changing the orientation of these items. Nor can a user of PDH or the UbiTable exempt certain tabletop items in the central workspace from being included by global actions, such as a workspace rotation or view change. Users of the UbiTable system, however, can move an object out of the central workspace into their personal workspaces to exclude it from being included in global rotations.

While several design criteria map directly onto the roles of orientation they support (e.g., providing free rotation supports both the ease of reading and alternate perspective roles) there is some subtle interplay between others as well as other design factors which influence the roles of orientation. For example, while the ConnecTable addresses all design criteria except allowing user-overrides, its small, tiled display makes it difficult for users to simultaneously maintain personal and group spaces. Its form factor, consisting of 
two connected personal displays, favors multiple personal spaces, especially since the physical seam between displays is located in the natural centre of the group space. The connected displays also prevent a person from interacting with objects in a collaborator's display space, inhibiting intentional communication in this area.

Similarly, the form factor of the Café Table limits its ability to completely address the roles of orientation. It provides a small, semi-circular display that, besides the scrolling peripheral icons mentioned above, provides only a fixed group orientation. Because of its limitations for supporting collaboration, the Café Table interface is currently being redesigned on a larger table called the Opportunistic Browsing Table (Shen et al., 2004), which will likely address some of its original restrictions.

In contrast to the Cafe Table, both the InfoTable and InteracTable systems provide large, seamless workspaces that allow people to maintain personal and group spaces. The InfoTable also allows users to move objects between their laptop displays and the table display, providing an extended personal space for independent work while allowing easy sharing of items when appropriate.

Evaluating PDH against the roles of orientation highlights some fundamental assumptions underlying its design. PDH provides users with a large "group" workspace. The global orientation mechanisms, such as its LazySusan and Magnet rotations, provide workspace-wide orientation changes. These changes may interfere with residual effects of a rotation action, such as indication of ownership and establishment of a personal space. This approach assumes that story-sharing is a continually tightly-coupled activity, where people never work independently. This assumption seems unrealistic given the variety of collaboration styles that people often employ during group work (Cockburn and Greenberg, 1996).

To address this issue, the UbiTable system provides separate personal spaces on the table surface, as well as private spaces on nearby laptops. Although a personal workspace can be used to clearly indicate ownership, global rotations applied to the central workspace on the UbiTable may interfere with group members' ability to use orientation to indicate temporary ownership of items in the group space (as noticed in our observational study). Therefore, the UbiTable only partially satisfies the ability of orientation to be used to convey availability information. Furthermore, the automatic rotation provided by the passing mechanism in both PDH and the UbiTable makes it difficult for groups to use a single "group orientation" in the central workspace. As a result, the ability of group members to use orientation to convey support for an idea in the workspace - an important collaborative mechanism observed by Tang (1991) - is appreciably hindered. However, this passing mechanism provides support for orienting items for one's personal use and towards a particular person at the table for communicative purposes. 
Systems that attempt to address orientation by using multiple copies of information (e.g., ConnecTable, InteracTable) focus on the comprehensive role of orientation, but in so doing can compromise the coordinative and communicative roles. In particular, using an object's orientation to help indicate personal or group spaces or the ownership of specific objects is considerably degraded when multiple copies of the same object exist. Likewise, using an object's orientation for intentional communication or to speak to individuals without additional comments or gestures is appreciably hindered without the shared focus inherent to a single set of objects.

In summary, this comparison highlights several useful and versatile approaches to rotation and orientation, such as lightweight, free rotation gestures. It also emphasizes several deficiencies in current tabletop systems for supporting the multiple facets of orientation. In particular, more user control is needed in systems which provide automated support for orientation. While these systems can facilitate interaction, it is difficult for the computer to predict the intentions of the user and, thus, may interfere with the collaborative process unless flexible automatic and manual rotation capabilities are provided.

\section{Providing fluid orientation}

In creating an interaction mechanism to better support the three roles of orientation, we have used the following as design guidelines: the implications stated in Section 8, advice from kinesthetic studies involving rotation and translation (Wang et al., 1998), and a review of tabletop interface literature (Scott et al., 2003). Kinesthetic studies of the manipulation of physical objects have demonstrated that rotating and translating are inseparable actions in the physical world (Wang et al., 1998). Integrated interaction techniques are more appropriate for manipulating integrated attributes, and separable interaction techniques are more appropriate for manipulating separable

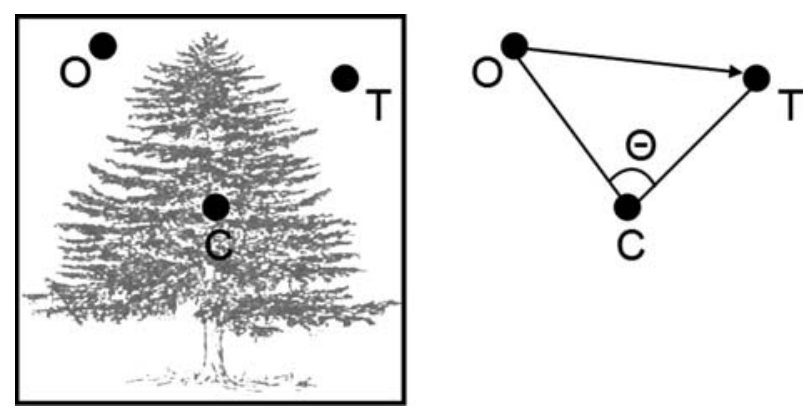

Figure 8. Three required points determine the RNT algorithm: $C$ - the $C$ enter of the object; $O$ - the Original mouse position; and $T$ - the new touch position (or Target). 
attributes (Jacob et al., 1994). Taking these two results together, an interaction technique that allows tabletop items to be simultaneously rotated and translated may be most appropriate for manipulation of these integrated attributes. An important secondary goal for tabletop interaction techniques is platform and technology independence. Collaborative interfaces for tabletop displays are still actively being researched, and it is unclear that any one tabletop system will be suitable for all types of collaborative tasks (Scott et al., 2003). Thus, developing interaction mechanisms that require specialized technology (for instance, multi-touch interaction) may place premature limits on desirable tabletop configurations. To provide integrated control of rotation and translation and better support for the three roles of orientation, we developed a rotation mechanism called Rotate 'N Translate (RNT).

RNT allows an object to be simultaneously rotated and translated in a single fluid motion, controlling $x$ and $y$ position plus orientation $(\theta)$ using a single $2 \mathrm{D}$ contact-point. There are three points of interest for determining the algorithmic behavior of RNT: $C$ - the Center of the object; $O$ - the $O$ riginal mouse position or contact-point, and $T$ - the new mouse position or Target. Figure 8 represents the case where the initial contact-point is located near the upper-left corner and movement is slightly down and to the right. In this case, the object is translated by the vector $O T$ and rotated about point $O$. The angle of rotation is the angle $\theta$, formed by $O, C$ and $T$. Note that the figure shows a large distance between $O$ and $T$; however, since the algorithm processes at 30 frames per second, in practice, the vector $O T$ tends to be only a few pixels long. After translation and rotation changes are applied, the original target point $(T)$ becomes the new original mouse position $(O)$; point $C$ remains the center position of the object, and a new target point $(T)$ is determined by the next directional movement.

An evaluation of RNT (Kruger et al., 2004) indicates that integrated control of rotation and translation does provide better support for all three roles of orientation than the common separated or moded interaction mechanism. For instance, the intentional communicative role of orientation, where an individual rotates and moves an object towards a collaborator to initiate communication, appears to be more fully supported with an integrated, free rotation mechanism (Kruger et al., 2004).

\section{Conclusions}

The main contribution of this paper is to expand our understanding of the roles that orientation plays in collaboration. The principle roles are:

- comprehension,

- coordination, and

- communication. 
We saw that orientation plays a significant role in the establishment and maintenance of personal and group workspaces. This, in turn, helps inform collaborators about who is currently using which items, and which items are available for group use. We also saw that a partial rotation towards a fellow collaborator is a particularly compelling communicative gesture that invites immediate collaboration. Finally, the long-term effect of the orientation of various items communicates to collaborators about the history of their work processes.

The second contribution of this paper is a set of design criteria for the design and evaluation of tabletop systems implied by this improved understanding of the roles of orientation. The third contribution is the use of these combined insights to develop a new integrated orientation and rotation technique, RNT, for tabletop displays. RNT allows an object to be simultaneously rotated and translated in a single fluid motion, using a single $2 \mathrm{D}$ contact-point. It provides clear feedthrough of rotation actions, indicates item position and orientation throughout the action, and maintains the orientation of rotated items when the manipulation is complete. By using only a single touch-point, RNT supports one-handed interaction and incurs minimal overhead for producing rotation. As well, RNT is technologyindependent - it can be used with both indirect and direct input systems, and requires no specialized hardware.

Research into applying this improved understanding of the three roles of orientation is on going. The resulting design guidelines have further implications about the integration of support for orientation in collaborative interfaces. For instance, the development of other global interface approaches to orientation is planned and further investigations into the effectiveness of these techniques are underway.

\section{Acknowledgements}

This research was supported in part by the Natural Sciences and Engineering Research Council of Canada (NSERC), Alberta Informatics Circle of Excellence (iCORE) and Intel Inc. We thank Tony Tang for editing help and, more generally, the members of the Interactions Lab for their insightful comments. We would also like to thank anonymous reviewers of previous versions of this paper for their discerning comments which have helped shape and refine this work.

\section{References}

Brinck, T. and L. Gomez (1992): A Collaborative Medium for the Support of Conversational Props. In J. Turner and R. Kraut (eds.): $C S C W$ '92. Proceedings of the ACM Conference on 
Computer-Supported Cooperative Work, Toronto, Canada, October 31-November 4, 1992. New York: ACM Press, pp. 171-178.

de Bruijn, O. and R. Spence (2001): Serendipity Within a Ubiquitous Computing Environment: A Case for Opportunistic Browsing. In G. Abowd, B. Brumitt and S. Shafer (eds): Ubicomp '01. Proceedings of the Conference on Ubiquitous Computing, Atlanta, Georgia, September 30-October 2, 2001. New York: Springer, pp. 362-370.

Clark, H. (1996): Using Language. Cambridge: Cambridge University Press.

Cockburn, A. and S. Greenberg (1996): Children's Collaboration Styles in a Newtonian Microworld. In M.J. Tauber (ed): CHI '96. Conference Companion of the ACM Conference on Human Factors in Computing Systems, Vancouver, Canada, April 13-18, 1996. New York: ACM Press, pp. 181-182.

Deitz, P. and D. Leigh (2001): DiamondTouch: A Multi-User Touch Technology. In B. Schilit (ed.): UIST '01. Proceedings of the ACM Symposium on User Interface Software and Technology, Orlando, Florida, November 11-14, 2001. New York: ACM Press, pp. 219-226.

Fitzmaurice, G., R. Balakrishnan, G. Kurtenbach and B. Buxton (1999): An Exploration into Supporting Artwork Orientation in the User Interface. In M. Williams, M. Alton, K. Ehrlich and W. Newman (eds.): CHI '99. Proceedings of the ACM Conference on Human Factors in Computing Systems, Pittsburgh, Pennsylvania, May 15-20, 1999. New York: ACM Press, pp. 167-174.

Guiard, Y. (1987): Asymmetric Division of Labor in Human Skilled Bimanual Action: The Kinematic Chain as a Model. The Journal of Motor Behavior, vol. 19, no. 4, pp. 486-517.

Hall, E. (1966): Distances in Man: The Hidden Dimension. Garden City, New York: Double Day.

Hancock, M. and K. Booth (2004): Improving Menu Placement Strategies for Pen Input. GI'04. Proceedings of the 2004 Conference on Graphics Interface, London, Ontario, Canada, May 17-19, 2004. Waterloo, Ontario, Canada: Canadian Human-Computer Communications Society, pp. 221-230.

Jacob, R., L. Sibert, D. McFarlane and M. Preston Mullen, (1994): Integrality and Separability of Input Devices. ACM Transactions on Computer-Human Interaction (TOCHI), vol. 1, no. 1 (March 1994), pp. 3-26.

Kruger, R. and S. Carpendale (2003): Exploring Orientation on a Table Display, Department of Computer Science, University of Calgary. 2003-726-29.

Kruger, R. and S. Carpendale (2002): The e-Table: Exploring Collaborative Interaction on a Horizontal Display, Department of Computer Science, University of Calgary. 2002-714-17.

Kruger, R., S. Carpendale and S. Greenberg (2002): Collaborating over Physical and Electronic Tables. In C. Neuwirth and T. Rodden (eds.): CSCW '02. Conference Supplement for the ACM Conference on Computer-Supported Cooperative Work, New Orleans, Louisiana, November 16-20, 2002. New York: ACM Press, pp. 139-140.

Kruger, R., S. Carpendale, S. Scott and S. Greenberg (2003): How People Use Orientation on Tables: Comprehension, Coordination, and Communication. In M. Pendergast, K. Schmidt, C. Simone and M. Tremaine (eds.): GROUP '03. Proceedings of the ACM Conference on Supporting Group Work, Sanibel Island, Florida, November 9-12, 2003. New York: ACM Press, pp. 369-378.

Kruger, R., S. Carpendale, T. Tang and S. Scott (2004): Fluid Orientation on a Tabletop Display: Integrating Rotation and Translation, Department of Computer Science, University of Calgary: 2004-747-12.

Luff, P., C. Heath and D. Greatbatch (1992): Tasks-in-Interaction: Paper and Screen Based Documentation in Collaborative Activity. In J. Turner and R. Kraut (eds.): $C S C W$ ' 92. Proceedings of the ACM Conference on Computer-Supported Cooperative Work, Toronto, Canada, October 31-November 4, 1992. New York: ACM Press, pp. 163-170. 
Magerkurth, C., R. Stenzel and T. Prante (2003): STARS - A Ubiquitous Computing Platform for Computer Augmented Tabletop Games. In J. Scott (ed): UbiComp '03. Video Track and Adjunct Proceedings of the Conference on Ubiquitous Computing, Seattle, Washington, October 12-15, 2003. New York: ACM Press, pp. 267-268.

Pinelle, D., C. Gutwin and S. Greenberg (2003): Task Analysis for Groupware Usability Evaluation: Modeling Shared-Workspace Tasks with the Mechanics of Collaboration. ACM Transactions on Computer-Human Interaction, vol. 10, no. 4, pp. 281-311.

Rekimoto, J. and M. Saitoh (1999): Augmented Surfaces: A Spatially Continuous Work Space for Hybrid Computing Environments. In M. Williams, M. Alton, K. Ehrlich and W. Newman (eds.): CHI'99. Proceedings of the ACM Conference on Human Factors in Computing Systems, Pittsburgh, Pennsylvania, May 15-20, 1999. New York: ACM Press, pp. 378-385.

Ringel, M., K. Ryall, C. Shen, C. Forlines and F. Vernier (2004): Release, Relocate, Reorient, Resize: Fluid Techniques for Document Sharing on Multi-User Interactive Tables. In E. Dijkstra-Erickson and M. Tscheligi (eds.): CHI '04. Extended Abstracts of the ACM Conference on Human Factors in Computing Systems, Vienna, Austria, April 24-29, 2004. New York: ACM Press, pp. 1441-1444.

Scott, S., K. Grant, and R. Mandryk. (2003): System Guidelines for Co-Located, Collaborative Work on a Tabletop Display. In K. Kuutti, E. Karsten, G. Fitzpatrick, P. Dourish, and K. Schmidt (eds.): Proceedings of ECSCW '03 (Helsinki, Finland, September 2003), Dordrecht, The Netherlands: Kluwer Academic Publishers, pp. 159-178.

Scott, S., R. Mandryk and K. Inkpen (2002): Understanding Children's Collaborative Interactions in Shared Environments. Journal of Computer-Aided Learning, vol. 19, no. 2, pp. 220-228.

Shen, C., K. Everitt and K. Ryall (2003): UbiTable: Impromptu Face-to-Face Collaboration on Horizontal Interactive Surfaces. In A. Dey, A. Schmidt and J. McCarthy (eds.): UbiComp '03. Proceedings of the Conference on Ubiquitous Computing, Seattle, Washington, October 12-15, 2003. New York: Springer, pp. 281-288.

Shen, C., N. Lesh, B. Moghaddam, P. Beardsley and R. S. Bardsley (2001): Personal Digital Historian: User Interface Design. In J. Jacko and A. Sears (eds.): CHI '01. Extended Abstracts of the ACM Conference on Human Factors in Computing Systems, Seattle, Washington, March 31-April 5, 2001. New York: ACM Press, pp. 29-30.

Shen, C., N. Lesh, F. Vernier, C. Forlines and J. Frost (2002): Building and Sharing Digital Group Histories. In C. Neuwirth and T. Rodden (eds.): CSCW '02. Proceedings of the ACM Conference on Computer-Supported Cooperative Work, New Orleans, Louisiana, November 16-20, 2002. New York: ACM Press, pp. 324-333.

Shen, C., F. Vernier, C. Forlines and M. Ringel (2004): DiamondSpin: An Extensible Toolkit for Around-the-Table Interaction. In E. Dijkstra-Erickson and M. Tscheligi (eds.): $C H I$ '04. Proceedings of the ACM Conference on Human Factors in Computing Systems, Vienna, Austria, April 24-29, 2004. New York: ACM Press, pp. 167-174.

Stathis, K., O. de Bruijn and S. Macedo (2002): Living Memory: Agent-Based Information Management for Connected Local Communities. Interacting with Computers, vol. 14, no. 6, pp. 663-688.

Streitz, N., J. Geißler, T. Holmer, S. Konomi, C. Müller-Tomfelde, W. Reischl, P. Rexroth, P. Seitz and R. Steinmetz (1999): i-LAND: An Interactive Landscape for Creativity and Innovation. In M. Williams, M. Alton, K. Ehrlich and W. Newman (eds.): CHI '99. Proceedings of the ACM Conference on Human Factors in Computing Systems, Pittsburgh, Pennsylvania, May 15-20, 1999. New York: ACM Press, pp. 120-127.

Streitz, N., P. Tandler, C. Müller-Tomfelde and S. Konomi (2001): Roomware: Towards the Next Generation of Human-Computer Interaction Based on an Integrated Design of Real 
and Virtual Worlds. In J.A. Carroll (ed.): Human-Computer Interaction in the New Millenium. Reading, MA: Addison-Wesley, pp. 553-578.

Tandler, P., T. Prante, C. Müller-Tomfelde, N. Streitz and R. Steinmetz (2001): ConnecTables: Dynamic Coupling of Displays for the Flexible Creation of Shared Workspaces. In B. Schilit (ed.): UIST '01. Proceedings of the ACM Symposium on User Interface Software and Technology, Orlando, Florida, November 11-14, 2001. New York: ACM Press, pp. 11-20.

Tang, J. (1991): Findings from Observational Studies of Collaborative Work. International Journal of Man-Machine Studies, vol. 34, no. 2, pp. 143-160.

Tse, E. and S. Greenberg (2002): SDGToolkit: A Toolkit for Rapidly Prototyping Single Display Groupware. In C. Neuwirth and T. Rodden (eds.): CSCW '02. Conference Supplement for the ACM Conference on Computer-Supported Cooperative Work, New Orleans, Louisiana, November 16-20, 2002. New York: ACM Press, pp. 173-174.

Vernier, F., N. Lesh and C. Shen (2002): Visualization Techniques for Circular Tabletop Interfaces. In M. De Marsico, S. Levialdi and E. Panizzi (eds.): AVI '02. Proceedings of the ACM Conference on Advanced Visual Interfaces, Trento, Italy, May 22-24, 2002. New York: ACM Press, pp. 257-263.

Wang, Y., C. MacKenzie, V. Summers and K. Booth (1998): The Structure of Object Transportation and Orientation in Human-Computer Interaction. In C.-M. Karat, A. Lund, J. Coutaz, and J. Karat (eds.): Proceedings of CHI '98 (Los Angeles, CA, April 1998), New York: ACM Press, pp. 312-319. 